\title{
BOUNDARY RIGIDITY FOR SOME CLASSES OF MEROMORPHIC FUNCTIONS
}

\author{
VLADIMIR BOLOTNIKOV
}

\begin{abstract}
Let $f$ be a function meromorphic on the open unit disk $\mathbb{D}$, with angular boundary limits bounded by one in modulus almost everywhere on the unit circle. We give sufficient conditions in terms of boundary asymptotics at finitely many points on the unit circle $\mathbb{T}$ for $f$ to be a ratio of two finite Blaschke products. A necessary condition is that $f$ has finitely many poles in $\mathbb{D}$, i.e., that $f$ is a generalized Schur function. Similar rigidity statements are presented for generalized Carathéodory and generalized Nevanlinna functions.
\end{abstract}

Mathematics subject classification (2000): 30E05.

Keywords and phrases: generalized Schur functions, boundary interpolation.

\section{REFERENCES}

[1] V. M. Adamjan, D. Z. Arov, And M. G. KreǏn, Analytic properties of the Schmidt pairs of a Hankel operator and the generalized Schur-Takagi problem, Mat. Sb., 86(128) (1971), 34-75.

[2] D. AlPAY, S. REICH AND D. SHOIKHET, Rigidity theorems, boundary interpolation and reproducing kernels for generalized Schur functions, Preprint, arXiv:0709.2504.

[3] A. C. Antoulas And B. D. O. Anderson, On the scalar rational interpolation problem: The scalar case, IMA. J. of Math. Control and Information, 3 (1986), 61-88.

[4] A. C. Antoulas And B. D. O. Anderson, On the stable rational interpolation problem, Linear Algebra Appl., 122-124 (1989), 301-329.

[5] A. C. Antoulas, J. A. Ball, J. Kang And J. C. Willems, On the solution of the minimal rational interpolation problem, Linear Algebra Appl., 137/138 (1990), 511-573.

[6] M. ARSLAN, Rigidity of Analytic Functions at the Boundary, arxiv.org/abs/math/0605026v2.

[7] J.A. BAll, I. Gohberg AND L. Rodman, Interpolation of Rational Matrix Functions, OT45, Birkhäuser-Verlag, Basel-Boston, 1990.

[8] L. BARACCO, D. Zaitsev And G. ZAMPIERI, A Burns-Krantz type theorem for domains with corners, Math. Ann., 336, 3 (2006), 491-504.

[9] V. Belevitch, Interpolation matrices, Philips Res. Rep., 25 (1970), 337-369.

[10] V. Bolotnikov, A uniqueness result on boundary interpolation, Proc. Amer. Math. Soc., 136, 5 (2008), 1705-1715.

[11] V. Bolotnikov, On boundary angular derivatives of an analytic self-map of the unit disk, C. R. Acad. Sci. Paris, Ser. I, 347 (2009), 227-230.

[12] V. Bolotnikov AND A. KheIfeTs, A higher order analogue of the Carathéodory-Julia theorem, J. Funct. Anal., 237, 1 (2006), 350-371.

[13] V. Bolotnikov AND A. Kheifets, Carathéodory-Julia type conditions and symmetries of boundary asymptotics for analytic functions on the unit disk, Math. Nachr., in press.

[14] F. BRACCI, R. TAURASO AND F. VLACCI, Identity principles for commuting holomorphic self-maps of the unit disc, J. Math. Anal. Appl., 270, 2 (2002), 451-473.

[15] D. BurnS AND S. G. KRANTZ, Rigidity of holomorphic mappings and a new Schwarz lemma at the boundary, J. Amer. Math. Soc., 7, 3 (1994), 661-676.

[16] D. Chelst, A generalized Schwarz lemma at the boundary, Proc. Amer. Math. Soc., 129, 11 (2001), 3275-3278. 
[17] M. Elin, M. Levenshtein, D. Shoikhet And R. TAuRaso, Rigidity of holomorphic generators and one-parameter semigroups, Dynam. Systems Appl., 16, 2 (2007), 251-266.

[18] M. G. KREINN AND H. LANGER, Über die verallgemeinerten Resolventen und die charakteristische Funktion eines isometrischen Operators im Raume $\Pi_{\kappa}$, Colloq. Math. Soc. János Bolyai, 5 (1972), 353-399.

[19] T. L. KRIETE AND B. D. MACCLUER, A rigidity theorem for composition operators on certain Bergman spaces, Michigan Math. J., 42, 2 (1995), 379-386.

[20] D. Shoik Het, Another look at the Burns-Krantz theorem, J. Anal. Math., 105 (2008), 19-43.

[21] R. TAuraso, Commuting holomorphic maps of the unit disc, Ergodic Theory Dynam. Systems, 24, 3 (2004), 945-953.

[22] R. TAURASO AND F. VLACCI, Rigidity at the boundary for holomorphic self-maps of the unit disk, Complex Variables Theory Appl., 45, 2 (2001), 151-165. 